\title{
Tourism and Travel can Effectively Benefit from Technologies Associated with Industry 4.0
}

\author{
Vasile Mazilescu \\ vasile.mazilescu@,ugal.ro \\ Dunarea de Jos University of Galati, Romania
}

\begin{abstract}
Connecting many products to the Internet, presence of sensors, wireless communications expansion, robot and intelligent machine development, real-time data analysis have the potential to transform how travel and tourism are made. The benefits of developing intelligent applications in the field of tourism consist in recognizing enabling technologies as a real catalyst in driving business efficiency for the coming years. This paper highlights the fact that Artificial Intelligence (AI), Internet of Things (IoT), Robotics, All Reality are really a major business topic, particularly in the field of tourism, and there is a sufficient capacity for these technologies to support future business travel models. We consider that the conceptual and practical evolution of Industry 4.0 can positively and consistently influence the development of tourism, and there are many similarities between them. Even though the concrete applications of the AI in tourism are still insufficiently elaborated, we believe that extremely rapidly, by increasing the capacity (mobility, speed, volume od data, the ubiquity of data processing and data transfers, interconnection) of intelligent processing, tourism and tourists will benefit from much closer services of concrete needs throughout travel.
\end{abstract}

Keywords: Industry 4.0, AI, IoT, All Reality

\section{Introduction}

The demand in the field of tourism is constantly increasing in the context of the global economy, which goes from the manufacturing form of production to the economy based on packages of services and knowledge, using information technologies in order to automate the different business processes. There has been an important demographic change in recent years: the generation of millennials has a much greater inclination for tourist activities (it uses technology intensively, presents specific needs for communication, consumption and tourist orientation), the third generation is continuously growing and having sufficient resources for tourism (with specific options and needs in terms of personalization, consumption of services, security and desired options), the middle class is extremely interested in increasingly complex tourism packages. New destinations appear required by the above population categories. Emerging markets will soon surpass developed markets in terms of international arrivals. Tourism is increasingly supported by the digital transformation involving all major states and companies (using mobile technologies, social media, smart platforms, long-term business plans) that continuously influence the entire sector. The lifestyle based on relaxation, socialization, health maintenance will become more and more important in making tourists' decisions and consequently in developing more and more complex tourist packages, easier to manage even by tourists through intelligent digital transformation, that is to say according to the dynamics of life. The services are highly personalized and tourists prefer direct interaction with the suppliers. There are preferences, interests and expectations that can be aggregated individually using dedicated computer technologies and with capabilities in flexible request resolution. The travel products requested are extremely heterogeneous. For this reason, tourism service providers can find their own niche segment in this growing market, helped by increasingly specialized online platforms. Digital transformation has both a horizontal and a vertical impact on the value chain. This implies that companies need to better integrate and digitize their vertical data flow, from 
product development and procurement to processing and transport logistics and it involves a horizontal collaboration with key suppliers, customers and other value chain partners, for example by using product identification and monitoring solutions $[5,30]$. For companies, these issues involve the creation of complex digital solutions. In addition, companies are developing new products and services with digital features that cover the entire life cycle of the product and therefore facilitate close contact with end-users [2]. Companies also invest in digital services and create complete solutions tailored to their customers' ecosystems, often in collaboration with partners in the value chain. Recent advances have made IC\&T more affordable and cheaper, thanks to Internet access, cloud development, and blockchain [6]. Thus, digital solutions have become available for smaller players and, more importantly, have become available to more market players. They have benefited from massive computerization and travel agencies, regardless of their size [9]. The entire hotel industry has gained access to global availability and booking systems. Virtually any accommodation unit can use these solutions. It is enough to analyze the development of Airbnb in order to observe the size of the phenomenon [1,23]. The tourism industry is a global sector with business opportunities available without regional restrictions. AI becomes extremely useful in this respect. AI's application possibilities in the travel and hotel industry are almost infinite, ranging from the hyper personalisation of services to intelligent equipment management, intelligent orientation, or automated editorial content production [3]. Even small companies can initiate a project that integrates AI, provided they demonstrate agility, implement appropriate management and have the necessary raw material, ie data and knowledge. AI technology faces a period of acceleration due to current advances in data and knowledge processing $[10,11,19]$. Personalization is one of the most spectacular aspects of its application in tourism: by learning what the customer is looking for, buying and wanting, AI can create tailor-made and personalized offers [14]. Chatbots, voice assistants, robots, real-time translation systems, augmented reality, all the interfaces supported by AI, should soon accompany the tourist in his walks. They are virtual assistants and banks who say that AI has begun to integrate the business travel sector and vice versa. Technology evolves rapidly and allows these tools to improve every day, especially with regard to personalizing the services offered to the traveler. Emerging technologies, such as IoT, VR and AI, will help people identify and act on their information without emotional interference or external subjective ideas, while helping them to exert their human reasoning, where appropriate [8]. In the short term, we will be witnessing AI applications in hiring and promotion procedures, their role being to filter intentional and unintentional subjectivism. The VR is used as an interrogation tool, facilitating the provision of merit-based opportunities. In the future, all businesses will be technological business, and our free time will turn into an interconnected experience [4].

The present paper consists of five sections. Section 2 attempts to make a series of connections between Industry 4. 0 and travel/tourism industry, which can be developed in line with all current and future achievements of Industry 4.0. Section 3 is a general outllook of present and future emerging technologies that have a tremendous potential in remodeling business processes in the tourism industry. Section 4 analyzes the applicability of intelligent technologies in the tourism industry, more precisely how it will be in categorically influenced this industry by the major changes of the semantic Web. Section 5 highlights a few conclusions and future research directions in this highly attractive and influential field of business in relation to the evolution megatrends of emerging technologies, including AI.

\section{Characteristics of present and future Tourism Industry}

Travel packages are a complex web-based online booking system specially designed for travel management companies, for destination management companies. They are intended for various travel deals: B2B travel agencies, B2B2B distributors, B2B2C booking system, B2B2C corporate booking system, travel agent and travel agencies, customized travel portal (travel site) solution for each client according to its requirements and budget [7]. The travel portal is a booking solution for flights, hotel holidays, cars, buses, transfers and travel agent activities to book customers, 
receive commissions, and set margins for bookings. Travel agents can manage fund allocations, policies, branches, suppliers, part of the booking flight, and payment management. Tourism is primarily a business-to-consumer business (B2C), with consumers as a target group of individuals. Companies offering intelligent travel and tourism applications seem to rely on existing ideas to attract small and medium-sized businesses and to acquire the data they need for the learning model and agent based systems development [13]. Tourism is based on mobile technologies. Demand and supply are typically met through Internet networks, which requires a great deal of cooperation and trust, that is, security. We have been in the digital age for some time and we are almost solving the details of our holidays by mail and on the internet, whether we organize them on our own or we ask for help from travel agencies. You pay and make the necessary bookings online, then you receive the specific codes by email. Transactions are secure and all transactions take place in real time. You check-in from any laptop or smartphone, and at the hotel you just provide the booking code and wait for the key or the card. Nowadays, there are electronic tickets that are scanned immediately at the airport before boarding. So get rid of the caution of printing tickets every time you fly. The same is true for hotel bookings, which you usually receive by e-mail in the form of a code that you have to present at the reception. Today, this process is much shorter using smart devices. The entire process of ordering, paying and traveling can be completed within minutes. Following the development of IC \& T systems, the tourism industry has changed dramatically and decision-making has accelerated. The new decision-making system (including Virtual Personal Assistant - VPN) is also beneficial to service providers because they can align to existing capabilities. AI systems can learn personal preferences and individual customer requirements better than travel agents. Online reservation systems in airlines have not only led to a better use of capabilities but also price comparability. The low growth of cost airlines has further forced industrial innovation and efficiency gains. Websites such as Expedia, Orbitz, or Kayak have made the decision process much easier for the consumer.

The Industry 4.0 concept is based on the adoption of enabling technologies such as Big Data, AI or IoT by traditional manufacturing industries using digital transformation, around people, data and processes business. Originally, leading-edge technologies were only available to large airlines, global distribution systems (GDSs) were not available to all market players. Most of the time, travel agencies only received terminal access to these systems. Industry 4.0 features that revolutionize it in the information technology era are summarized next: Interoperability (the ability of machines, devices, sensors, and people to connect to them through IoT or IoP Internet of People), Transparency of information (the ability of computer systems to create a virtual copy of the physical world by integrating sensors into business or industrial processes), Technical Assistance (the ability of support systems to aid people by aggregating and viewing comprehensive information for informed decision-making and resolving urgent issues in the shortest possible time as well as the ability of physical cybernetic systems to physically support people by performing a series of tasks that are unpleasant, too extenuating or unsafe for people), Decentralized decisions (the ability of physical cybernetic systems to make decisions on their own and to carry out their tasks as autonomously as possible using cross-solutions; the operator interaction is the same, regardless of the type of control system architecture/the particularities of the various factories are normalized by the Cloud database), Uniformity of information (the main benefit for the user and a basis for the company's economy), Modularity (the ability to quickly and seamlessly adapt to seasonal changes and market trends). All of these features make companies more flexible and receptive to business trends. They become more innovative, increase productivity and optimize production. Industry 4.0, by its characteristics, also requires the tourism industry to have the same characteristics, thus obtaining Tourism 4.0 [28].

Connecting many products to the Internet, the presence of sensors, the expansion of wireless communications, the development of robots and intelligent machines, the analysis of real-time data have the potential to transform the way the production is realized. Connecting the physical world with the virtual world in cyber-physical systems will have a disruptive impact on 
technologies, manufacturing processes and people. The exponential technological advance, manifested by the processing power, the storage capacity and the multitude of applications developed have made the industry evolve to this level. From the economic point of view Industry 4.0 represents a chance to relaunch the production and the evolution of the business models for services and products, as follows: the application of ICT to digitize the information and integrate systems to the conception, development, manufacture and use of the products; development of new software technologies for modeling, simulation, virtualization and digital manufacturing; development of cyber-physical systems to monitor and control physical processes; evolution of 3D printers and additive manufacturing to simplify manufacturing; improvement of decision support systems for human operators, the emergence of intelligent tools and assistance using AR; increasing the capabilities of human-machine interaction in a simpler and natural sense. In the future, a larger number of consumers and producers in the tourism sector will be digitally interconnected, which will lead to the highest level of personalization of products. Companies will be able to respond much faster to customer requirements by accessing real-time data. Devices, process control systems, production systems will be connected to IoT platforms. The sensors will be integrated with the production equipment to monitor their functionality and to predict when maintenance is to be carried out. The equipment will be repaired when necessary before making non-compliant products. In addition, machine state and productivity data can be recorded and transmitted in real-time to the cloud for statistical analysis of the data. This is particularly useful when it comes to reducing maintenance costs. Devices equipped with sensors could also be integrated into various business environments through VR and AR technology. In this way, we can display real-time processes through computer-generated sensory inputs such as movies or simulation models. Using AR together with real-time data availability in the business environment helps the staff involved in these activities, while providing clearer information about processes. AR and wireless systems should be used to share and view information through the cloud to help improve manufacturing and monitoring processes. With mobile and cloud applications, 3D print technology will continue to grow in the future. Significant sales growth is estimated for industrial equipment with sensors and actuators that can exchange data with other machines and computer networks in real-time via the cloud. Digitization is expected to double over the next five years. A significant number of companies are developing new products and services with digital features that cover the entire life cycle of the product. In a business environment where companies identify patterns and methods to improve their business, optimize their workflows and collaborate more efficiently, mobile technologies offer the increasingly demanding performance required by those more demanding players to ensure this goal is met. Companies will want to make better use of organizational data, keeping trends already known. Optimizing and managing multi-media environments is a priority for many companies, as well as the need to integrate security at a basic level. None of these trends will disappear, and we will see a mix of expanding and developing already evolving trends with a series of constantly changing requirements that force organizations to adopt digitization and new technologies at a faster pace. Innovations based on AI and ML have solved long-standing problems, leading to the belief that these technologies can be a solution to many challenges. Large volumes of network data are analyzed, security threats are identified, or uninterrupted workflows are provided. Cisco DNA Analytics can extract anonymous data from customer networks, identify patterns and provide statistics. Cost savings with network operation are thus obtained, and security is greatly improved. ML is used to find malware in encrypted traffic without decrypting it.

\section{A general outllook of Emerging Technologies that can sustain Tourism and Travel Development}

The travel industry includes all activities that help consumers reach their destination using different travel modes. Tourism refers to travelers, tourists travel for pleasure and visit places of interest such as museums, art galleries, theaters, national parks, religious sites, etc. The tourism industry includes all firms that provide goods or services to facilitate business or leisure activities 
in locations different from where we live. The tourism industry refers to people who travel for business or pleasure. Within the travel and travel industry there are different types of activities, managed by specialized companies. The transport sector is extremely important and covers activities based on air, rail, water or land. The air transport industry has grown enormously from all points of view [25]. Car rental, water or rail transport, space travel, accommodation, hotel industry, private accommodation, cruises, specialized tourism, food and beverage supply, entertainment clubs, casinos, shopping, all these activities are essentially sources business for the travel and travel industry $[15,17,26]$. Over the past decade, almost all aspects of how we work or live have become more and more digitized. Internet and mobile technologies have brought the first wave of digital transformation known as the Internet of People. The amount of information generated by the Web of Things (WoT) will be several times higher than the volume of information provided by the classic Internet. It is likely that in the short term, the AI growth potential will come from improving productivity. The development and application of AI, Big Data and Machine Learning (ML) methods in the tourism and hotel industry as mobile platforms have a major impact in personalizing services and supporting outstanding experiences for emerging generations by understanding customer behavior, quantifying events throughout the journey as well and the development of robotic recommendations or service packages in the field and related fields $[12,18]$. Increased labor productivity is expected to account for more than $50 \%$ of the contributions made to GDP by AI between 2018 and 2030 and, more generally, of emerging technologies of which AI belongs. We observe within the IC\&T specialized teams an increase in the interest of exploring emerging technologies [16,21,27]. We discuss about AI and ML applications, a lot of algorithms and statistical models that develop learning processes based on large data sets like Big Data, Internet of Things. We need Robots Process Automation (RPA), Advanced Analytics (including advanced statistical methods and ML being components of AI), $5 \mathrm{G}$ connectivity (the next standard of telephones mobile that will work with the Semantic Web and AI applications), Cloud Infrastructure which allows remote and secure remote access as one of the basic components of the Semantic Web. RPA includes automating routine tasks, improving employee skills, and relieving repetitive tasks so that they can focus on value-added activities. AI is able to identify different components of demand, to calculate in advance prices associated with packages of tourism services, to propose different action plans. Attempts are currently being made by using social media and the Web to analyze the emotions and the degree of customer satisfaction. Natural Language Processing (NLP) and Fuzzy or Modal Logics methods are involved in these processes. Most likely, intensive capital intensive sectors, such as production or transport, will record the highest gains due to AI, as many of their operational processes are prone to automation. GDP growth, as a result of product improvement and further changes in demand, consumer behavior and consumption, all due to AI, will outweigh the rise in productivity gains. The new technological innovations are also absorbed in the travel industry. Globalization will follow 4.0, which will affect the tertiary sector of services, so far untouched by the negative effects of globalization. The impact of the new industrial revolution will be transformative, both for the economic, social and political system. The physical world will become smaller compared to the new digital world interconnected [24]. The dematerialisation of the economy will accelerate through a transition to a circular economy favored by the new industrial revolution. Production will be permanently modified by automation, localization and customization, and the current supply chains will be replaced altogether. Large-scale autonomous agents such as robots, drones, and autonomous vehicles, intelligent software agents, use AI to automate the previously-fulfilled functions of people, especially high-repeatability or hostile activities $[22,29]$. Their automation goes beyond the automation provided by rigid programming models and exploits AI to provide advanced behaviors that interact more naturally with the environment and with humans. Enhanced analysis focuses on a certain area of augmented intelligence, using ML to transform how the content of analysis is developed, consumed and divided. Analytical capabilities will rapidly advance as a key feature of data preparation, data management, modern analysis, business process management, data mining-based processing platforms, and having inputs at the level of different large 
volume data volume transactions big data. Transparency of transactions must be a common goal of all companies involved in the development of peer-to-peer (P2P) block-based platforms, because only such an approach can enhance widespread adoption. Blockchain technology could significantly improve systems that support the development of smart cities where tourism can be deployed more effectively and more effectively in the context of Web 3.0. or Semantic Web. Traffic signs, traffic lights, traffic cars and other traffic components could be equipped with sensors that collect data and send them to a center that could establish routes that are as accessible to users as possible, a decongestion of traffic and a reduction of carbon emissions, could help in finding parking places, fueling stations or signaling accidents. The semantic web can be compared to an AI assistant that understands its user and personalizes everything. The purpose of the Semantic Web is to solve the boundaries of the traditional data-based Web by adding to the Web information a formal representation of its meaning in the form of explicitly represented knowledge. A direct benefit of semantics that can be processed by the computer is to increase the pace and automate information management tasks (searching or integrating data and distributed knowledge using logical inferences, developed in the context of various formal logical systems). AI should can transform our thinking on how economic growth is sustained [20].

\section{Applicability of Intelligent Technologies in the Tourism Industry}

In recent years, interest and investment in cognitive systems and AI have seen an explosive growth that will continue in both academic, scientific and technological development as well as commercial and business, from the smallest to the larger corporations. The most prominent names in technology (Google, Facebook, Microsoft, Yahoo) are investing heavily in AI that they embed in their own business models and increasingly use them in their offer: Virtual Assistants, Artificial Views, Speech Recognition, automatic translation and lots of other applications. All this is basically based on the technology of deep "artificial" neural networks and deep learning. One of the first areas in which they have been developed and in which they have gained widespread popularity is that of artificial vision, respectively recognition or classification of images. There is an important paradigm shift to initialize neural network parameters with values closest to optimal values using Restricted Boltzmann Machines (RBM). First, an unsupervised layered coat of the network is made for the weights to shape the intimate data structure. The initialized weights are adjusted using backpropagation. After that, was introduced instead of RBM auto-encoders, developed later with various variations such as denoising and self-encoding sparse. Later was introduced another algorithm for finding parameters, which achieved better results than before. We can see these results as a third wave of deep learning in the field of artificial neural networks, and in the last years they are experiencing an impressive revival and development, enhanced by the spectacular technological evolution in terms of capacities and speeds of computation and storage, use Graphics Processing Unit (GPU) for matrix parallel calculations, data acquisition devices, and the availability of larger and more diverse volumes of data (Big Data) in more and more areas [31]. New neural models and deep neural network architectures, inspired by either neuroscience or computational engineering, have been developed, deep learning technology nowadays virtually synonymous with $\mathrm{AI}$ and $\mathrm{ML}$ concepts. To understand the influences of $\mathrm{AI}$ on business processes, it must be seen as a physical capital-labor hybrid. AI can emulate work activities on a much larger scale and speed and may even perform certain tasks beyond the capabilities of the people. In some areas, AI has the ability to learn faster than people, even though the level of depth and analytical capacity differs from that of people, and more specifically from experts in different fields. AI aims to develop systems that can adapt to a concrete and dynamic business environment. In the learning phases, predictive algorithms make it possible to identify models or repetitive structures on large databases. This then allows the approximation of an unknown date to the known patterns or structures and then the qualification of a certain level of probability. Models or criteria must be identified to evaluate the ethical character of the algorithm used. For example, an algorithm used to determine the price of an insurance product from a large amount of information from a customer base discriminated against the customer's gender. In this case, we can renew the learning phase by removing the 
examination of this information. But this may implicitly lead to an equivalent bias, as a function of, for example, purchases made previously by the client.

The impact of AI on the tourism industry relates to services offered and customer engagement. In this regard, many of the available applications have developed chatbots modules that are a communication interface that helps individuals and companies to have successful conversations. It's actually an AI program that talks to the client and informs you about all the things you need to know. They are used to replicate important interactions with users, help business processes, obtain information from large groups, or simply be used as personal assistants. Search Engines use bots to probe the web and archive new pages for future hits. The first level is represented by any messaging platform: Facebook, Twitter, Slack, etc. The same principle applies to Amazon Alexa or Google Assistant or to any other consumer of webpages. The client connects to the bot either through a text channel or through a voice channel. In the case of voice option, there are two internal levels that deal with speech recognition and its transformation into input text and speech synthesis for responses. The NLU (Natural Language Understanding) component helps to extract the purpose of the discussion and entities from the user request. The NLU component consists of a supervised intentional classification model that is trained on a plurality of input sentences and the purpose of the target discussion, and a suitably trained entity extraction model. We expect a massive impact of deep-learning technologies - chatbots, virtual booking assistants (airline and hotel), travel-based AI-based travel assistance. The airline component of the tourism industry, being a highly concentrated industry with large companies, has developed its own technologies alongside classic IC\&T. With the emergence of these technologies in the commercial segments - Internet, TCP / IP protocols, cloud - IC\&T of the airline began to converge to them. As soon as a company has invested significant amounts in certain technology platforms, it is quite complicated to quickly align other technologies, even if they are newer and cheaper. Many companies have seen that the effort to give up their own legacy systems, even if they are outdated, is enormous. GDSs have developed global platforms based on mainframes. We talk about an enormous transactional power and high storage power, too (petabites data and transaction milestones), and migrating them without causing interruptions is an extraordinary challenge. One of the characteristics of the tourism industry is that it practically never stops, so changing systems is complicated and challenging, as are intelligent assistants based on voice recognition. Captured voice recordings are transcribed, annotated, and then reintroduced into the software as part of the effort to improve the way the virtual assistant works. This process of voice analysis is not devoid of the human side. Alexa lives in the cloud and always gets smarter. But, like many software built to learn from experience, people have an important role to play in this process. Some records prove to be troublesome or surprisingly aggressive. The security and privacy of the customers of each travel company must be taken seriously. Only an extremely small sample of voice recordings is enlightened to improve customer experience. Employees do not have direct access to information that can identify the person or account. All information is treated with great confidentiality and multiple authentication can be used to restrict access, service encryption, and auditing of the control environment. Virtual wizard settings give users the option to disable the use of voice recordings by the company to develop new features. Data analysis uses either client voice samples that are sent to analysts, but also transcripts of user orders, comparing records with automatic device transcription, analyzing user-machine interaction. In particular, employees check that the virtual assistant has provided an effective response to what the tourist has asked for. Others also note background conversations, and some details, such as banking data or people names, should be marked as critical. Emerging technologies and the way we use them can help define almost perfectly the categories of customers. We can not only find out, in real-time, what are their preferences and habits, we can also understand the sequence of actions in time and space so that we can define our most important contact points and ways of interaction and, of course, we can communicate with clients. Important applications in tourism industry are chatbots. A chatbot is a communication interface that helps individuals and companies to have successful conversations. One reason for 
the spread of chatbots is that many users are reluctant to use new applications. In addition to this, it is clear that it is not profitable to create, promote and make operational an application that will not be useful. By working on different platforms, chatbots solve this problem by using their multi-channel. Essentially, it is an AI program that communicates with the user. They are used to replicate important interactions with users to help business processes to get information from large groups. Or they can be used simply as personal assistants. Search Engines use bots to probe the web and archive new pages for future hits. Such an intelligent system is structured on several levels. The first level is represented by any messaging platform: Facebook, Twitter, Slack, etc. The client connects to the bot either through a text channel or through a voice channel. In the case of voice option, there are two internal levels that deal with speech recognition and its transformation into input text and speech synthesis for responses. The NLU (Natural Language Understanding) component, which helps to extract the purpose of the discussion and entities from the user request, consists of a supervised intentional classification model that is trained on a plurality of input sentences and the purpose of the target discussion, and a model extracting entities that can be pre-trained similarly to the Spacy model or StanfordNLP bookstores. This component produces bot responses. The easiest way to get this is by using a template mechanism. A more complex method is to use proposition generators via DL. Some of the major challenges of the hotel industry are competition differentiation, on-site recommendations, natural language communication with foreign clients, optimized prices, etc. The radical personalization will change this. Hotels will be able to offer unique personalized packages based on the person's interests, including multiple activities in one package as the client himself would do. By offering personalized packages, hotels no longer have to be competitive at the price level. Hotels start using algorithms of the next-product-to-buy type. These algorithms can analyze historical data to determine, for example, whether a customer prefers coffee in the morning, whether he / she takes his spouse on travel, and then using the location of the person's mobile phone to give a buy-one-receive - free when the customer enters the hotel's café in the morning. Hotels have begun to use such bots that can lead a tremendous customer experience that can suggest places of interest and that can make things happen by chat or voice. Because the system maintains the status, you can start the conversation on a channel (messenger, for example) and continue on another channel (email, Echo) without repeating it. Some hotels create a chatbot ecosystem that relates to customers before, during and after the trip. Some could use chatbot to check if a hotel is available. If customers have completed their booking, a chatbot can then send custom content links such as articles about top restaurants in the area, tourist guides, or other local exploration opportunities. Once booked, a shoe can allow customers to check in, request room service, order from the restaurant, and after check-out will collect feedback or ask them where they want to travel. The key to success lies in assuming customer experience from start to finish and maintaining the open communication channel. One of the greatest opportunities is the use of predictive analytical data and profound customization to optimize prices. For example, a hotel could have a surplus of rooms available due to a last-minute cancellation (a flight is canceled). To remedy this situation, the AI could reduce the price and promote this special price among locals.

\section{Conclusions}

The Industry 4.0 is becoming extremely important from the economic perspective due to already known causes such as globalization, technological development, consumer services, changing the demand structure, increasing mobility both at the technological and also at the people lavel. Revenues will grow in creative areas, such as the tourism industry, venture capitalists and those who will make the first moves in the new emerging markets. The Internet is expanding in ways we could not have imagined. Soon, more IP traffic will be generated than in all years since the launch of the internet. In less than three years, the global population will use the Internet, but new users will be greeted by an internet that connects people via IoT devices, home appliances and automated vehicles, that is, an Internet where mobility will increase substantially. Also, VR and AR traffic will increase over the next few years five times as more and more consumers and 
companies use these technologies. Mobile connectivity will continue to grow. Mobile data traffic will continue to grow relative to other types of traffic. Blockchain will be used in new and innovative ways. Blockchain will continue to expand in areas such as supply chain management, networking, digital identity, and currency transactions. Most likely, all major cloud service providers will implement Blockchain at the commercial level, and this will become a key element in most $\mathrm{AI}$ and IoT offers over the next few years. Companies will have to rethink their network. More and more companies will need to focus on network transformation to continue delivering premium customer experiences. Currently networks have not been built to meet network needs in the coming years. Whether it's 5G, VR, IoT or AI / ML, behind all these trends is a largecapacity digital network composed of a multitude of public and private networks. And because it is estimated that by 2020 one million items will be connected to the Internet every hour, this infrastructure is under great pressure. Manually managing networks becomes impossible. Companies must now be able to turn on any device, anywhere, at any time, regardless of the domain. The new architecture will have to be automated, anticipate actions, block security threats and continue to evolve and learn. The IoT will generate a huge amount of data and so you will need very high speeds for transfer and processing. IT companies will have a high involvement, Industry 4.0 will attract new suppliers for cyber-physical systems (CPS - CyberPhisical System) or services: IT security, Big Data analysis, M2M and AI solutions. Data management and security will be key issues to solve. To realize the true potential of Industry 4.0, companies need to plan for digital transformation. Companies need to understand the importance and urgency of digitalization, both success and failure will happen at very high speeds. We need in the future strong technologies that can contribute to economic growth based on the maturation of many concepts and issues related to automation of work, monitoring, verification, interpretation, decision making, recommendation, prediction, learning.

\section{References}

1. Airi Lampinen and Coye Cheshire (2016). Hosting via Airbnb: Motivations and financial assurances in monetized network hospitality. InProceedings of the 2016 CHI Conference on Human Factors in ComputingSystems (CHI '16). ACM, 1669-1680.

2. Borràs, Joan, Antonio Moreno, and Aida Valls (2014). Intelligent Tourism Recommender Systems: A Survey. Expert Systems with Applications 41 (16): 7370-7389, Elsevier, bttps:// romisatriawahono.net/lecture/ rm/survey/information\%20retrieval /Borras\%20\%20Tourism\%20Recommender\%20Systems\%20-\%202014.pdf

3. Boztas, S. (2017). Automated holidays: how AI is affecting the travel industry, The Guardian, SustainableBusiness, www.theguardian.com/sustainable-business/2017/feb/17/ holidays-travel-automatedlastminute-expedia-skyscanner

4. Bubalis, D., \& Leung, R. (2018). Smart hospitality-Interconnectivity and interoperability towards an ecosystem. International Journal of Hospitality Management, 71(April), 41-50. DOI: 10.1016/j. ijbm.2017.11.011, https://mmw.researchgate.net /publication/322209171_Smart_hospitalityInterconnectivity_and_interoperability_towards_an_ecosystem

5. Chatzisavva Panagiota (2017). Digital Transformation in tourism sector, International Hellenic University, bttps:/ / repository.

ihu.edu.gr/xmlui/ bitstream/ handle/11544/29204/Dissertation_Chatzisavva_Panagiota.pdf?sequence=1

6. Chuang, T. C., Liu, J. S., Lu, L. Y. Y., Tseng, F.-M., Lee, Y., \& Chang, C.-T. (2017). The main paths of eTourism: trends of managing tourism through Internet. Asia Pacific Journal of Tourism Research. bttps:/ / doi.org/10.1080/10941665.2016.1220963

7. Cognizant (2017). The Future of Air Travel: Eight Dismptive Waves of Change, bttps:/ / www.cognizant.com/whitepapers/ the-future-of-air-travel-eight-dismuptive-waves-of-changecodex2566.pdf

8. David Hanes, Gonzalo Salgueiro, Patrick Grossetete, Robert Barton, Jerome Henry, (2017). IoT Fundamentals: Networking Technologies, Protocols, and Use Cases for the IoT, 
bttp://ptgmedia.pearsoncmg.com/images/9781587144561/samplepages /9781587144561_CH08.pdf, Cisco Press 800 East 96th Street Indianapolis, Indiana 46240 US A

9. Eide, D., Fuglsang, L., Sundbo, J. (2017). Management challenges with the maintenance of tourism experience concept innovations: Toward a new research agenda. Tourism Management, 63, 452-463. bttps:/ / doi.org/10.1016 /j.tourman.2017.06 .029

10. Gajdošik, T. (2019). Towards a Conceptual Model of Intelligent Inf. System for Smart Tourism Destinations. In R. Silhavy (Ed.), Software Engineering and Algorithms in Intelligent Systems (pp. 66-74). Springer, Cham. DOI: 10.1007/978-3-319-91186-1_8.

11. Hao, J. X., Yu, Y., Law, R. \& Fong, D. K. C. (2015). A genetic algorithm-based learning approach to understand customer satisfaction with OTA websites. Tourism Management. bttps:/ / doi.org/10.1016/j.tourman.2014.11.009

12. IBA Global Employment Institute (2017). Artificial Intelligence and Robotics and Their Impact on the Workplace

13. Juan Liu, Chaobui Wang, Shimin Fang Tingting Zhang (Christina) (2019). Scale development for tourist trust toward a tourism destination, Tourisme Management Perspectives, Volume 31, pp. 383-397, bttps:// doi.org/10.1016/j.tmp.2019.07.001

14. KIBO Software Inc. (2017). The ultimate Guide to personalization. Discover the four phases of personalization to supercharge customer experiences. bttps://wnw.websitemagazine.com/docs/defaultsource/Whitepapers/kibo-theultimateguideto perso nalization.pdf?sfursn $=0$

15. KMPG \& FCM, (2019). Redefining Corporate Travel Management Pillars for effective managed travel, Pillars for effective managed travel, https:// wnw.fcomtravel.com/sites/default/files/201902/FCM_Redefining_Corporate_Travel_Management_2019.pdf

16. Logesh, R., Subramaniyaswamy, V., Vijayakumar, V., Gao, X.-Z.,\& Indragandhi, V. (2017). A bybrid quantum-induced swarm intelligence clustering for the urban trip recommendation in smart city. Future Generation Computer Systems. bttps:// doi.org/10.1016 /j.future.2017.08.060

17. Matlack, C. (2017). An army of Chinese millennials is reshaping global travel. Bloomberg Businessweek. Retrieved June,2018, wnw.bloomberg.com/news/articles/2017-11-02/an-army-of-chinese-millennials-isreshaping-global-travel

18. Moniz, A.B. (2015). Intuitive Interaction Between Humans and Robots in Work Functions at Industrial Environments. In The Role of Social Robotics. In Social Robots from a Human Perspective; Vincent, J., Taipale, S., Sapio, B., Lugano, G., Fortunati, L., Eds.; Springer: Heidelberg, Germany, 2015; pp. 6776, bttps:/ / link.springer.com/ chapter/10.1007\%2F978-3-319-15672-9_6

19. NTG Skills Alliance, (2019). Desk Research Summary on the Future of Digital, Green and Social Skills in Tourism, bttps:/ / nexttourismgeneration.eu/ upcontent/ uploads/2019/03/NTG_Desk_Research_Summary_January_2019.pdf

20. Or, M. (2018). AI meets human hospitality. Who will run our hotels in 25 years -Humans or Robots? White Paper IDeaS Revenue Solutions and SiteMinder. https://downloads.siteminder.com/en/ai-meetsbuman-hospitality-white-paper.pdf

21. Pizam, A. (2017). The Internet of Things (IoT): The next challenge to the hospitality industry. International Journal of Hospitality Management, $62 \quad$ (April), $\quad$ pp. 132-133 bttps:/ / doi.org/10.1016/j.ijhm.2017.02.002

22. Qian, F., Han, C., \& Haiyan, M. (2016). Intelligent model system for tourism flow prediction. In Proceedings of the 2016 International Conference on Intelligent Information Processing -ICIIP '16. bttps:/ / doi.org/10.1145/3028842.3028845

23. Quinby, D. (2016). The End of Alternative Accommod.: Airbnb is Now the Third-Largest OnlineAccommodation Seller Worldwide, Phocuswright,www.phocuswright.com/Travel-Research/ResearchUpdates/2016/The-End-of-Alternative-Accommodation.

24. Skift Report (2018). 10 Business Travel Trends for 2019, bttp:/ / veilletourisme.s3.amazonaws.com/2019/01/10-Business-Travel-Trends-for-2019.pdf

25. Shaolong Sun, Yunjie Wei, Kwok-Leung Tsui, Shouyang Wang, (2019). Forecasting tourist arrivals with machine learning and internet search index, Tourism Management 70, http://iranarze.ir/wpcontent/uploads/2018/09/E9411-IranArze.pdf 
26. Stacey, J. (2015). Supporting quality jobs in tourism. OECD Tourism Papers, 2015/02. Paris, France:OECD Publishing. Retrieved September,2018, http:/ / dx.doi.org/10.1787/5js4rugg7sqr-en

27. Steve Mann, Tom Furness, Yu Yuan, Jay Iorio, and Zixin Wang, (2018). All Reality: Virtual, Augmented, Mixed (X), Mediated (X,Y), andMultimediated Reality, bttps:// arxiv.org/pdf/1804.08386.pdf

28. Urške Starc Peceny, Jurij Urbančic, Simon Mokorel, Vesna Kuralt and Tomi Ilijaš (2019). Tourism 4.0: Challenges in Marketing a Paradigm Shift, IntechOpen, https:// cdn.intechopen.com/pdfs/65836.pdf

29. VALORIA, (2018). Barometrul digitalizării. Studiu despre percepția managerilor cu privire la impactul digitalizării asupra companiilor din România. https://valoria.ro/wpcontent/uploads/2018/05/Studiu_Barometrul_digitalizarii_2018_RO.pdf

30. Vuorikari, R., Punie, Y., Carretero Gomez. S., Van den Brande, G. (2016). DigComp 2.0: The Digital Competence Framework for citizens. Update phase 1: The conceptual reference model. Luxembourg: Publication Office of the European Union

31. Wee CH (2017). Emerging Trends in Tourism: Opportunities, Challenges and Implications. J Tourism Hospit 6: 318. doi: 10.4172/21670269.1000318 